\title{
Session 7: Molecular biology - I
}

\author{
Thursday 9 October 2003. Moderators: Jim Larrick and Eskil Soderlind
}

\section{[14.15-14.45]}

\section{Optimisation of antibody therapeutics by phage and ribosome display \\ Andrew Buchanan, George Thom, Celia Shorrock, Lutz Jermutus and Ralph Minter \\ Cambridge Antibody Technology, Cambridge, UK}

Both phage display and ribosome display systems can be used effectively to isolate large numbers of sequence-diverse antibodies from naïve libraries. Once leads have been identified the same display technologies can be used to engineer the characteristics of the drug candidates to yield the desired in vivo efficacy. Ribosome display has a number of advantages over phage display resulting in significantly shorter timelines for therapeutic drug development. Using case studies we will demonstrate how ribosome display can effectively optimise antibodies to different types of targets. We will also highlight the ability of ribosome display to effectively explore the sequence space and its high throughput capacity.

\section{[14.45-15.15]}

Generation of improved protein therapeutics by directed evolution: Anti TNF- $\alpha$ and anti CD20 antibodies with increased potency

Dave Marquis, Elaine Conner, Brian Ondek, Eric Smith, Barrett Allen, Alain P. Vasserot and Jeffry D. Watkins

Applied Molecular Evolution, 3520 Dunhill Street, San Diego, CA 92121, USA

Directed evolution is a broadly applicable protein engineering technology that is ideally suited to enhance the therapeutic potential of biologics. The ability to ameliorate virtually any characteristic of a protein can translate into significant clinical benefits including, higher potency, greater efficacy, decreased immunogenicity and better safety profile. This approach was used to create two novel antibodies with improved functions and optimized therapeutic properties.
AME-527 is an anti TNF- $\alpha$ monoclonal antibody developed for the treatment of inflammatory diseases. The variable regions are composed of fully human frameworks designed to support CDRs specifically engineered to optimize the TNF- $\alpha$ neutralization properties of the antibody. AME-527 demonstrated superiority to infliximab, an antibody currently marketed for the treatment of Crohn's disease and rheumatoid arthritis. In direct comparisons, AME-527 showed a 15-fold increase in binding affinity and exhibited a 10-fold higher capacity to neutralize TNF- $\alpha$ in cell-based assays. In a transgenic murine model of polyarthritis that closely resembles human rheumatoid arthritis, these improvements translated into significantly higher potency, as measured by the inhibition of disease progression.

AME-133 is a humanized and optimized anti CD20 monoclonal antibody in pre-clinical development for the treatment of non-Hodgkin's lymphomas. Upon binding to its target, the antibody mediates the death of CD20 positive cells by mechanisms that involve effector functions. Consequently, the antibody was engineered to bind to CD20 with higher affinity and to promote more efficient destruction of tumor cells via enhanced binding to the Fc stimulatory receptor, CD16. In ex vivo ADCC assays using human effector cells, AME-133 exhibited equivalent tumor cell killing at $>$ 10-fold lower antibody concentrations compared to rituximab, a currently marketed anti CD20 therapeutic antibody.

Additionally, our approach to humanization resulted in antibodies with fully human, germline frameworks that may reduce the risk of immunogenicity when compared to the currently marketed chimeric molecules. The pre-clinical data collected on these two therapeutic antibody candidates indicate that they may have superior clinical properties relative to infliximab and rituximab and demonstrate the potential benefits of optimizing biotherapeutics. 


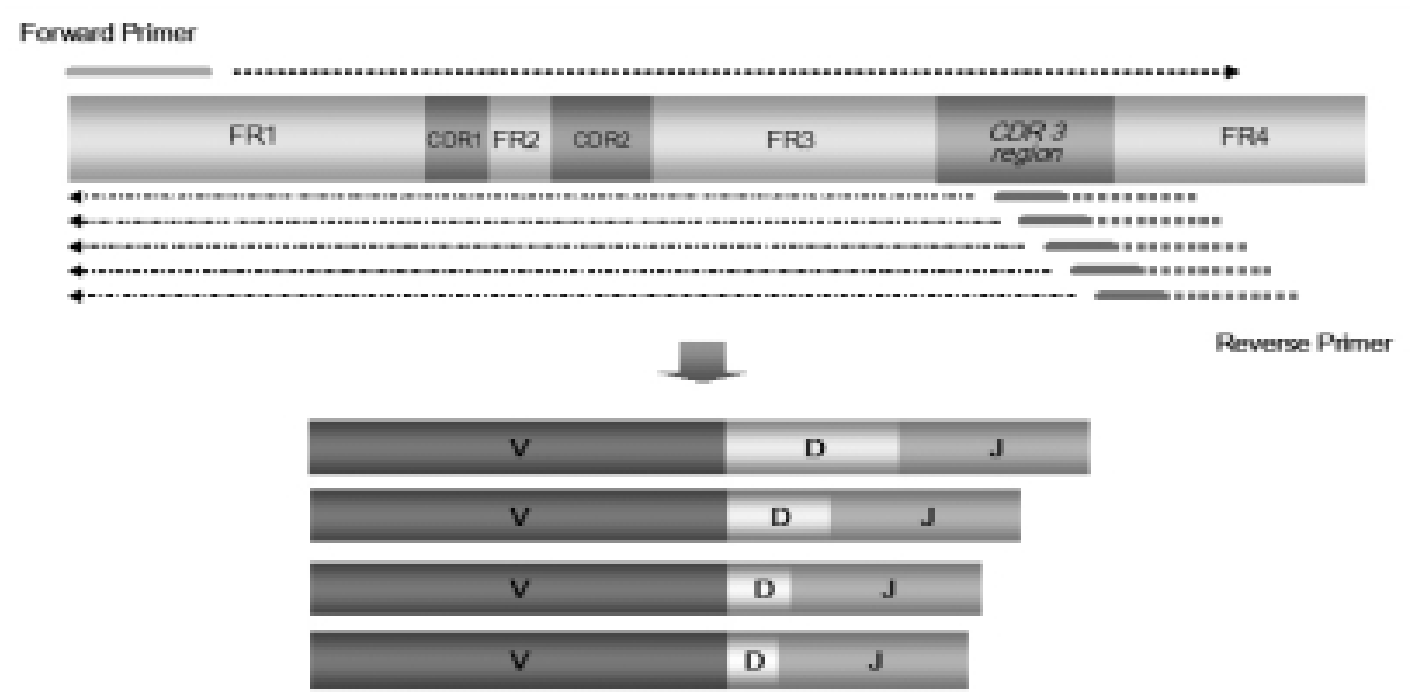

Fig. 1. Schematic diagram of frame-shifting PCR. Derived Ig genes variable region by semi-nested PCR were served as template for frame-shifting PCR which allows sliding of reverse primer along the CDR3 region of Ig gene variable region. After frame-shifting PCR modificatoin, a library of Ig variable region with different length and sequence within the CDR3 region has been generated.

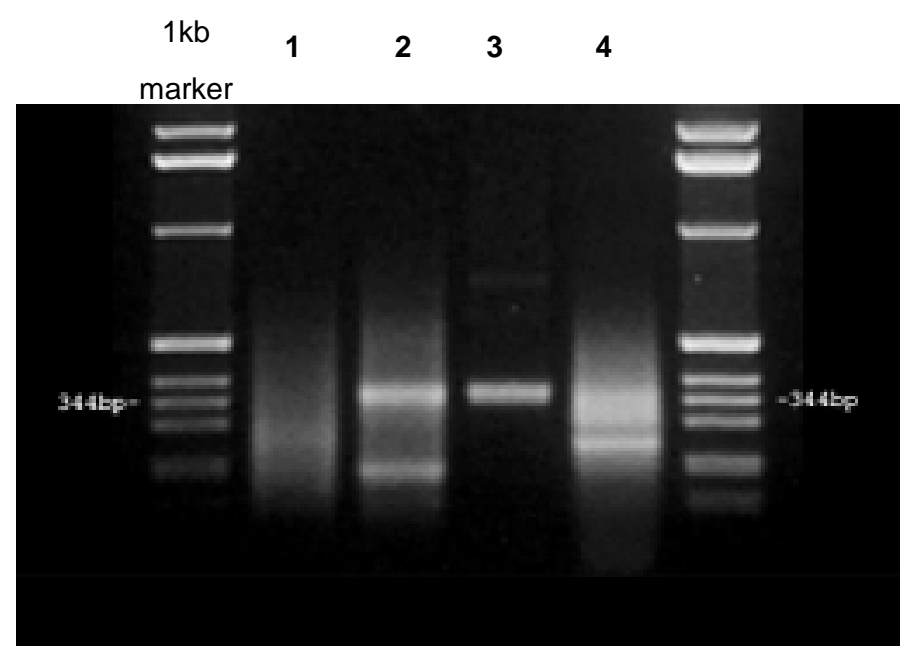

Fig. 2. Semi-nested PCR amplification of Ig heavy chain variable region. The PCR product of genomic DNA amplification (lane 1), semi-nested PCR amplification (lane 2), gel-extracted fragments of semi-nested PCR product (lane 3), PCR product after frame-shifting modification (lane 4). (Similar result of $\kappa$-light chain variable region is not shown here).

[15.15-15.30]

One step germline immunoglobulin genes retrieval and diversity enhancement for $\mathrm{ScFv}$ library

\section{construction}

Man Cheng and Wing-Tai Cheung *

Department Of Biochemistry, The Chinese University

Of Hong Kong, Shatin, N.T. HKSAR

E-mail: \{wtcheung, chengman\}@cuhk.edu.hk

${ }^{*} T e l .:+85226096104$

Introduction: Owing to the broad application and wide perspective of monoclonal antibody (mAb), important advances in design, selection, and production of engineered antibodies have been made. Traditional method for recombinant mAb construction like hybridoma technology has many limitations, such as duration, stability and class manipulation [1]. Although new technology like the display of antibody fragments on the surface of filamentous phages and the subsequent selection of antibodies have been proved as an effective tool for the isolation of antigen specific anti- 
(3a)

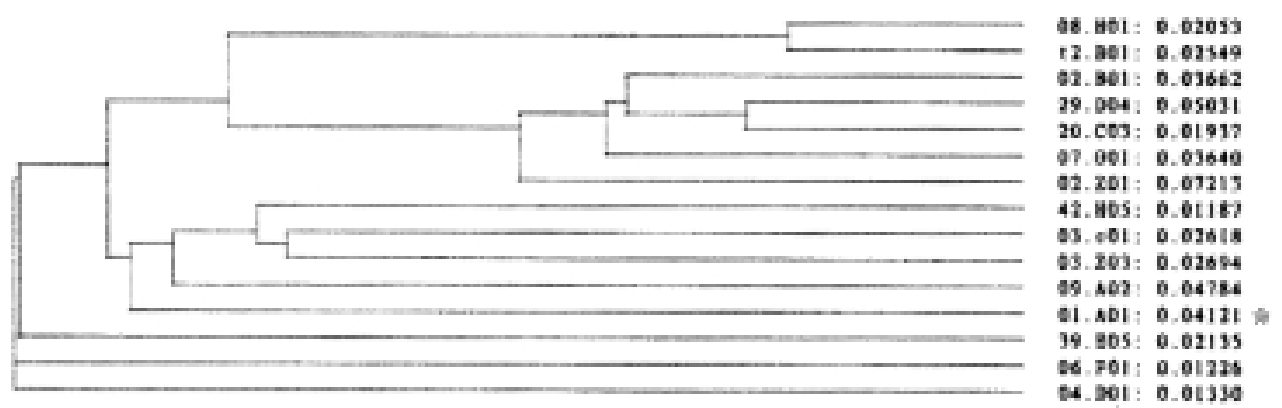

(3b)

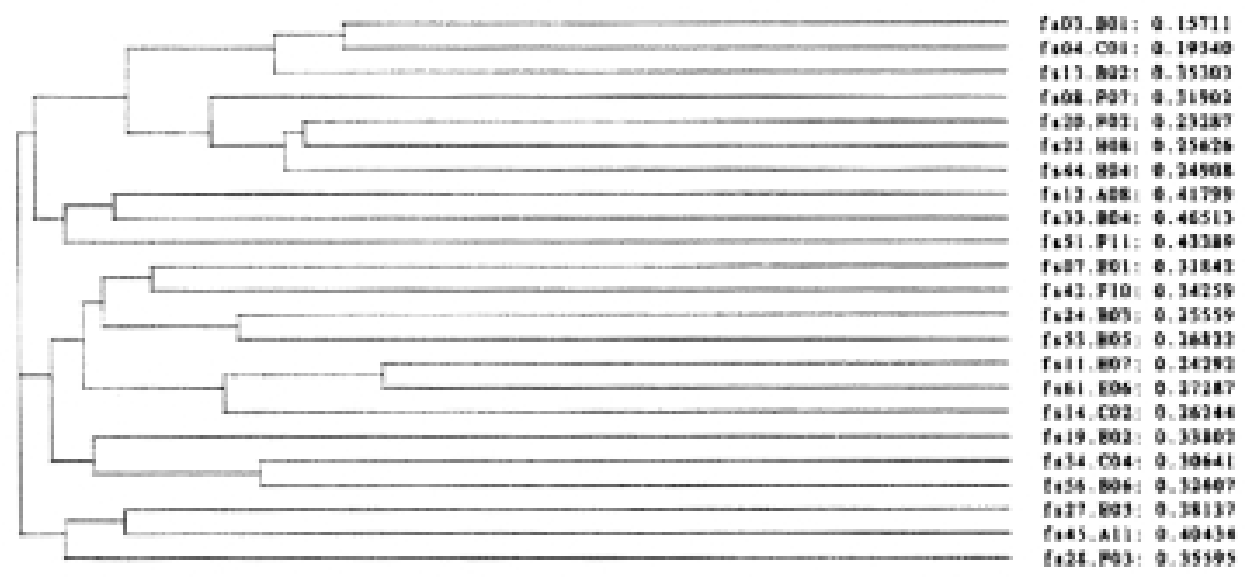

Fig. 3. Dendrogram of sequencing analysis of kapa-light (3a) and heavy (3b) chain variable region. The difference between the most related gene sequence is $23.6 \%$ and $62.3 \%$ for $\mathrm{V}_{\mathrm{L}-\kappa}$ and $\mathrm{V}_{\mathrm{H}}$ respectively.

\begin{tabular}{|c|c|c|c|}
\hline & CDALI & CDRL2 & CPRL3 \\
\hline 966 & 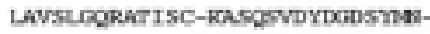 & -Aassivs & 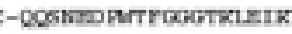 \\
\hline 2 & Easosvidycosma & Aassies & COSKEDLPRSACUNK \\
\hline 2 & reAsgsvirocosme & AAsELS & QQSEDOPAHSARAP \\
\hline 3 & EASgSWDYODSnA & AASELES & Cosknorsarned \\
\hline 4 & EASgSVDYDCDSna & AAstaLES & COEКTDRARSARER \\
\hline 5 & KASQSVDYDODSn & AASULBS & QQGBSORKYVRFWH \\
\hline 6 & RASESVDS-ONSFA & AAsQUES & QQGIBDORAVVRCWHR \\
\hline & $\cdots \cdots+\cdots$ & otentatet & 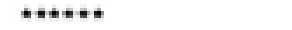 \\
\hline
\end{tabular}

Fig. 4. Frame-shifting PCR verification: amino acid sequence alignment of $\kappa$-light chain variable region of Ig genes derived from immunized mouse splenocytic genomic DNA. 5G6, the full sequence of Mus musculus 5G6 monoclonal antibody kappa light chain variable region, was used as reference Ig $\mathrm{V}_{\mathrm{L}-\kappa}$ sequence. Sequences \#1 to \#6 were frame-shifted $\mathrm{V}_{\mathrm{L}-\kappa}$ genes, which were members of the 01 .A01 Ig $\mathrm{V}_{\mathrm{L}-\kappa}$ family (Fig. 3(a) - marked with *), suggesting diversity-enhancement within the CDR3 region.

bodies [2-4], it still has its limitations.

In general, the affinity of isolated antibodies is proportional to the initial size of the library used for selection. Using mRNA as an enriched source of expressed and spliced antibody genes neglects the allelic exclusive genes so as half of the potential genes. Hence, the diversity of the library used for selection becomes limited. Moreover, gene loss will happen in both self-intolerance genes elimination and gene inactivation during maturation of B-lymphocytes. Finally, the other problem needs to be considered is the existence of non-functional genes. These genes refer to the $\mathrm{Ab}$ genes containing stop codon(s) in their segments, either naturally or created by the vague rearrangement 

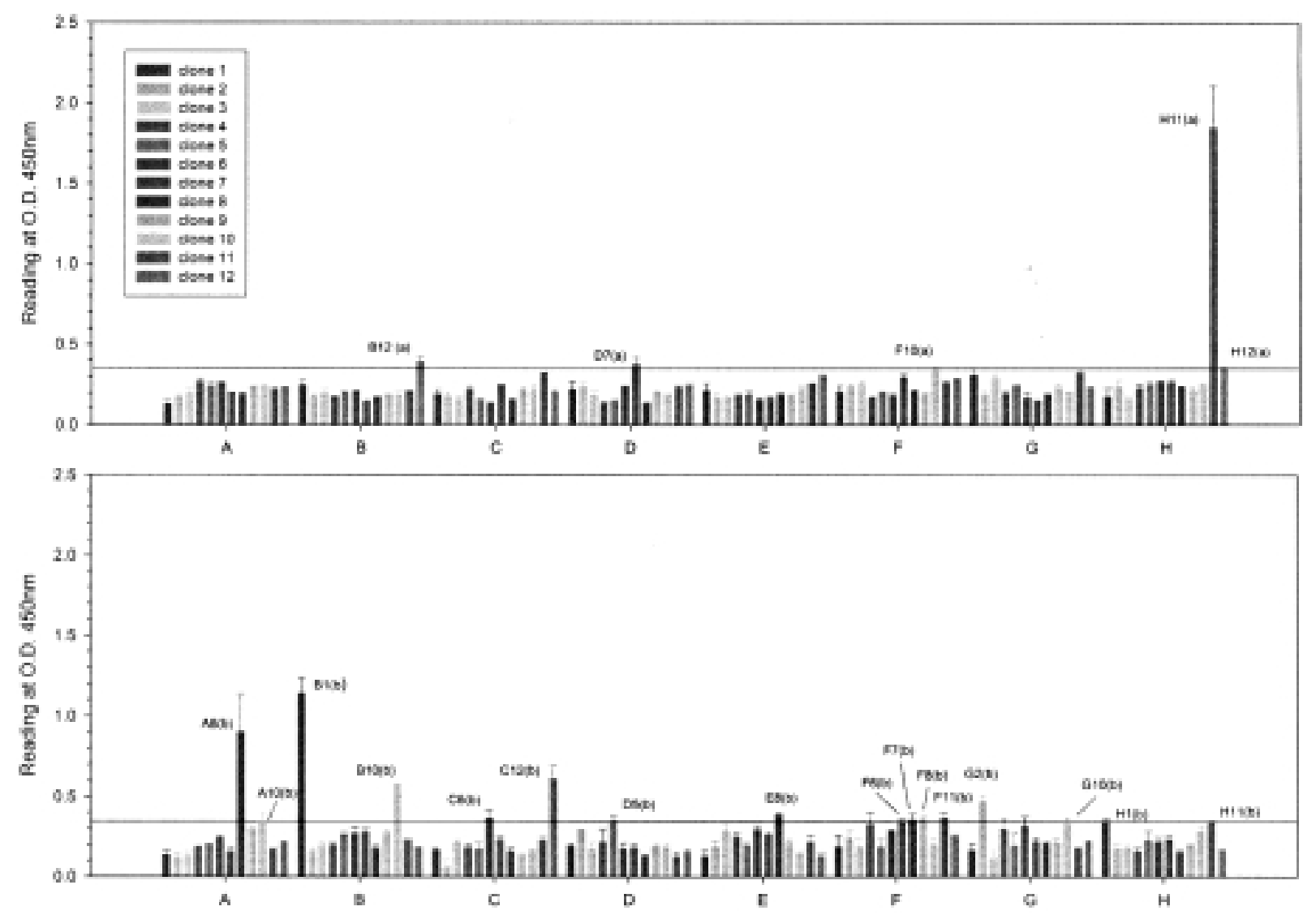

Fig. 5. PhageELISA of candidate clones obtained after five rounds of panning against phOx-BSA conjugate (partly). Clones with phageELISA reading more than 1.5 fold of the mean value of the sample set were isolated and subjected to further analysis. (K-S Prob. $<0.001$ ).

\begin{tabular}{|c|c|c|c|c|c|c|}
\hline & & COPEA & & $\cos =$ & & crsa3 \\
\hline Lastic & QusVIn-sctusarang & Drne & 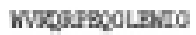 & 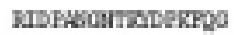 & 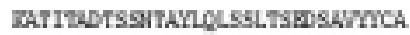 & JanoptsLTYSS \\
\hline $\operatorname{Lxac}$ & cusvesctusordux & prong & WVIORFEQGLENG & MTDFDODIRANKRPG & 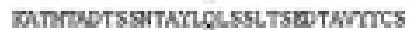 & OCWFELSLSL \\
\hline Lxc12: & cusvisectusorenx & Drmar & WVORFPEQTLENT & MTDFWODTARAKRPG & 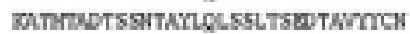 & ALESEIYYMRLR \\
\hline $\operatorname{tsesc}$ & GusvisectasGrux. & wrine & พVXอVIEQGLENC & 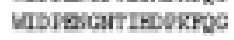 & 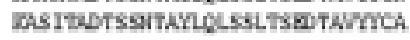 & SULTARUEGCOGTDLTVSS \\
\hline LSCAC & CAsvinectasGrax & Drmet & WVXUNEQGLEVG & 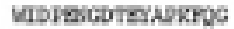 & 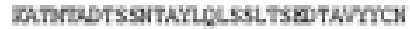 & AVFDG-PLVCWLESEASABCLLL \\
\hline LACA & assvesctusorenx & Drines & 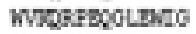 & BIDPQGOMTSODEKRA9 & BarInDTssmanqusstrsDoramca & RSTUATPGCONDEGTIVTVSA \\
\hline & 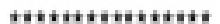 & & 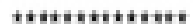 & 78 & 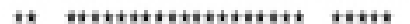 & \\
\hline
\end{tabular}

Fig. 6. Amino acid sequence alignment of heavy chain variable regions of isolated phOx-spcific ScFv. Alignment result of different members in a $\mathrm{ScFv}$ family indicated significant sequence variations within the CDR3 region.

processes during somatic recombination.

Results: This experiment describes a novel method for retrieving Ig genes from germline DNA as an alternative instead of using mRNA as the source for $\mathrm{mAb}$ preparation. A set of degenerated primers, which cover most of Ig genes, was used. The variable regions of immunoglobulin heavy and $\kappa / \lambda$-light chains $\left(\mathrm{V}_{\mathrm{H}} \&\right.$ $\mathrm{V}_{\mathrm{L}-\kappa / \lambda}$ ) were recovered from $\mathrm{CD}^{+} 19$ lymphocytic genomic DNA by semi-nested PCR method. Moreover, a supplementary PCR strategy ("frame-shifting PCR"), which mimicks somatic recombination, was used to in- troduce diversity into the CDR3 region of immunoglobulin so that recovers defective Ig genes resulted from non-productive exon joining and further enhances the diversity in the CDR3 region (Fig. 1).

As illustrated in Fig. 2, after genomic DNA amplication, variable regions of germline Ig genes were obtained by semi-nested PCR amplification (lane 1 to 3), which were further subjected to "frame-shifting PCR" to generate diverse Ig genes of variable length and sequence within the CDR3 region (lane 4). A library of either $\mathrm{V}_{\mathrm{L}-\kappa}$ or $\mathrm{VH}$ genes was obtained by cloning the 

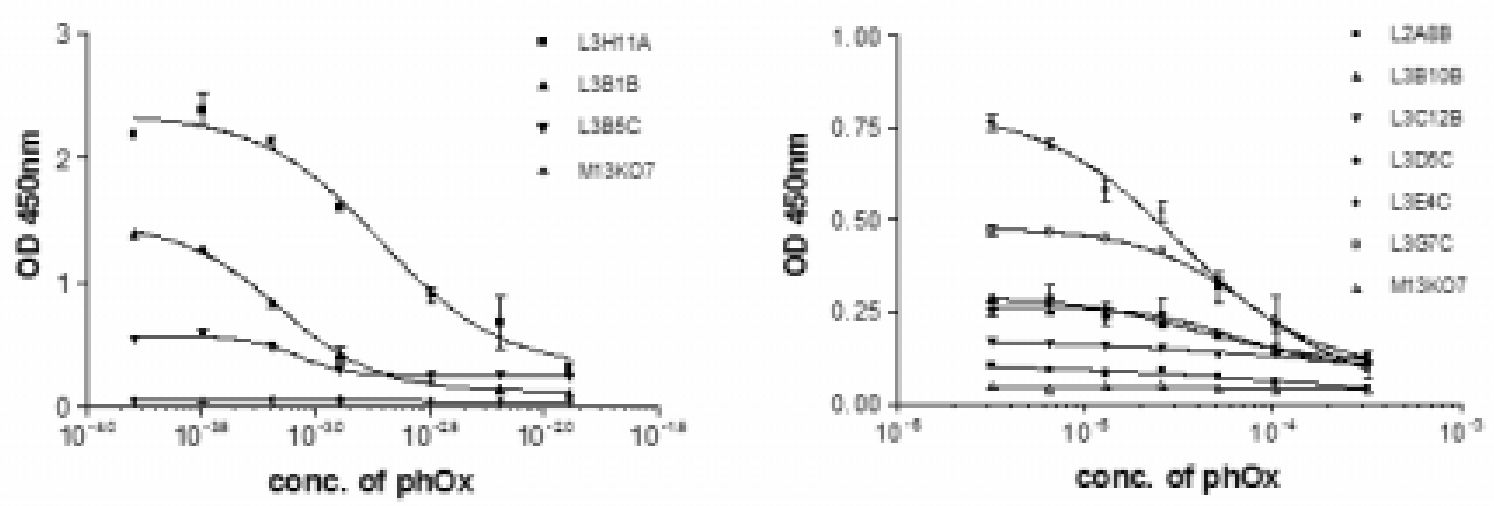

Fig. 7. Result of competitive phageELISA among high affinity (7a) and low affinity (7b) phOx-specific clones with the use of phOx-BSA conjugate as free ligand.

PCR fragments into TOPO TA cloning vector. Transformants (120) from each library were randomly picked for sequencing analysis. Phylogenic analysis indicated that 35 and 44 completely sequenced $\mathrm{V}_{\mathrm{L}-\kappa}$ or $\mathrm{V}_{\mathrm{H}}$ clones can be classified into 15 and 23 different families respectively (Fig. 3). Moreover, multiple sequence alignments revealed that significant sequence differences within the CDR3 region among members of a family (Fig. 4).

Feasibility of applying current method for preparation of antigen-specific antibody has been evaluated by constructing a small $\mathrm{ScFv}$ phage display library (5.16 $\times 10^{5}$ recombinants). A Balb/C mouse was immunized with 4-ethoxymethylene-2-phenyl-2-oxazolin-5one (EM-phOx) conjugated to chicken serum albumin (CSA) and its splenocytic CD19 ${ }^{+}$cells were used for the retrieval of Ig gene variable regions for library construction. After 5 rounds of panning against phOx conjugated to bovine serum albumin (BSA), potential candidate clones $\left(9.7 \times 10^{5}\right.$ recombinants) were identified. Clones (288) were randomly picked and their reactivities against phOx were determined by phageELISA. Forty-four highly reactive clones, of which reactivity towards EM-phOx were 1.5-fold higher than the mean value of the sample set, were isolated and further analyzed (Fig. 5). Phylogenic analysis of the sequences suggested that the derived Ig genes could be grouped into different classes and significant sequence variations were found within the CDR3 region of Ig genes in each class (Fig. 6). Furthermore, with the use of phOxBSA conjugate as free ligand, competitive phageELISA indicated significant differences in affinity among different clones (Fig. 7).

Conclusion: This approach offers a fast and simple way to retrieve the variable region of $\mathrm{Ig}$ genes and introduces sequence diversity in the CDR3 region simultaneously.

\section{References}

[1] Harlow and Lane, Monoclonal antibody lab. protocal, 1994, pp. 141-147.

[2] C.F. Barbas, A.S. Kang, R.A. Lerner AND S.J. Benkovic, Assembly of combinatorial antibody libraries on phage surfaces: the gene III site, Proc. Natl. Acad. Sci. USA 88 (1991), 7978-7982.

[3] J.D. Marks, By-passing immunization: building high affinity human antibodies by chain shuffling, Bio/Technology 10 (1992), 779-783.

[4] A. Nissim, Antibody fragments from a 'single pot' phage display library as immunochemical reagents, EMBO J. 13 (1994), 692-698. 